\title{
Endoscopic ultrasound-guided fine-needle aspiration vs fine-needle biopsy for the diagnosis of pancreatic neuroendocrine tumors
}

두(1) $(9$

\author{
Authors \\ Leonardo H. Eusebi ${ }^{1,2}$, Douglas Thorburn ${ }^{1}$, Christos Toumpanakis ${ }^{3}$, Leonardo Frazzoni ${ }^{2}$, Gavin Johnson ${ }^{1,4}$, Sheida \\ Vessal ${ }^{1}$, Tu Vinh Luong ${ }^{5}$, Martyn Caplin³ ${ }^{3}$ Stephen P. Pereira ${ }^{4,6}$
}

Institutions

1 HPB endoscopy unit, Royal Free Hospital NHS Trust, London, UK

2 Department of Medical and Surgical Sciences, University of Bologna, Italy

3 Neuroendocrine Tumour Unit, Centre for Gastroenterology, Royal Free Hospital NHS Trust, London, UK

4 Department of Gastroenterology, University College London Hospitals NHS Foundation Trust, London, UK

5 Department of Cellular Pathology, Royal Free London NHS Foundation, London, UK

6 Institute for Liver \& Digestive Health, University College London, UK

submitted 30.1.2019

accepted after revision 3.6.2019

Bibliography

DOI https://doi.org/10.1055/a-0967-4684 |

Endoscopy International Open 2019; 07: E1393-E1399

(c) Georg Thieme Verlag KG Stuttgart · New York

eISSN 2196-9736

Corresponding author

Prof. Stephen P. Pereira, The UCL Institute for Liver \&

Digestive Health, NW3 2PF, UK

Fax: +4407729632540

stephen.pereira@ucl.ac.uk
ABSTRACT

Background and study aims Endoscopic ultrasoundguided fine-needle aspiration (EUS-FNA) as a method of obtaining preoperative diagnosis of pancreatic neuroendocrine tumors (PanNETs) has been reported in several series. Fine-needle biopsies (FNB) are increasingly employed to obtain core specimens during EUS. However, the differences in efficacy between these sampling methods in the diagnosis of PanNETs still needs to be defined.

Patients and methods Over a 13-year period, all patients who underwent EUS-guided tissue sampling of suspicious pancreatic lesions with clinical, endoscopic and pathologic details were entered into an electronic database. Lesions underwent EUS-FNA or FNB sampling, or a combination of the two. The accuracy and safety of different EUS-guided sampling methods for confirmed PanNETs were investigated.

Results A total of 91 patients (M/F: 42/49, median age: 57 years), who underwent 102 EUS procedures had a final diagnosis of PanNET. Both EUS-guided sampling modalities were used in 28 procedures, EUS-FNA alone was used in 61 cases, while EUS-FNB alone in 13 cases. Diagnostic yield of EUS-FNA and EUS-FNB alone, including the inadequate specimens, was $77.5 \%$ (95\%Cl: $68.9-86.2 \%)$ and $85.4 \%$ (95\% Cl: 74.6-96.2\%), respectively. The combination of both sampling modalities established the diagnosis in $96.4 \%$ of cases $(27 / 28)$ (95\% Cl: $89.6-100 \%)$, significantly superior to EUS-FNA alone $(P=0.023)$. Diagnostic sensitivity among the adequate samples for EUS-FNA, EUS-FNB and for the combination of the two methods was $88.4 \%(95 \% \mathrm{Cl}$ : $80.9-96.0 \%$ ), $94.3 \%$ (95\%Cl: $86.6-100 \%$ ) and $100 \%(95 \%$ $\mathrm{Cl}$ : 100-100\%). There was one reported complication, a post-FNA bleeding, treated conservatively.

Conclusions EUS-FNB improves diagnostic sensitivity and confers additional information to cytological assessment of PanNETs. 

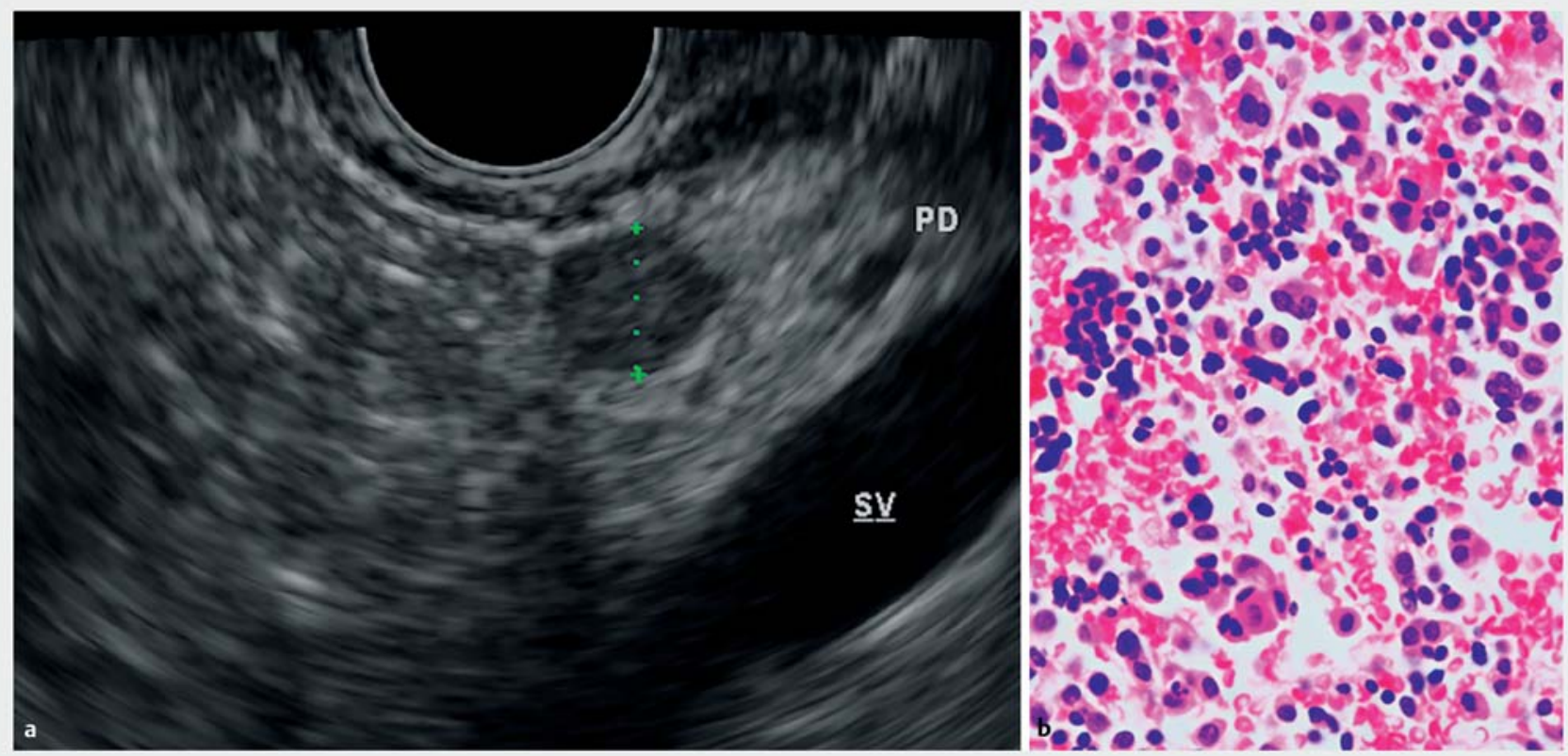

- Fig. 1 a EUS image of hypoechoic well-defined pancreatic NET and $\mathbf{b}$ histological pattern of a well-differentiated pancreatic neuroendocrine tumor.

\section{Introduction}

Pancreatic neuroendocrine tumors (PanNETs), despite having been steadily increasing in both incidence and prevalence during the past few decades, are rare ( 1 per 100,000 population), representing $1 \%$ to $2 \%$ of all pancreatic neoplasms [1,2]. Preoperative diagnosis is important since a solitary small tumor without evidence of metastatic spread may be suitable for pancreatic preserving surgery, such as enucleation, rather than more extensive resection [3, 4]. However, preoperative localization can be difficult, as these tumors are frequently smaller than $2 \mathrm{~cm}$ in diameter. Consequently, conventional imaging methods such as transabdominal ultrasound (US), computed tomography (CT), and magnetic resonance imaging (MRI) may fail to accurately localise the tumor in $10 \%$ to $40 \%$ of patients $[2,5,6]$. Similarly, somatostatin receptor scintigraphy has a limited sensitivity of $62 \%$ to $86 \%$ as well as false-positive rate of up to $12 \%$ [6].

Endoscopic ultrasound (EUS) has been reported to be highly accurate for preoperative localization of PanNETs, mainly primary insulinomas, which are frequently negative on SRS, and was established as an alternative to more invasive methods such as angiography. PanNETs are identifiable by EUS in $79 \%$ to $95 \%$ of suspected cases [7-9], and appear hypoechoic, round and homogenous, though they may occasionally be isoechoic or hyperechoic with irregular margins ( $\mathbf{F i g . 1}$ ) [10]. EUS-guided fine-needle aspiration (EUS-FNA) can confirm the diagnosis cytologically and provide information to guide the type of surgical intervention, which is particularly useful in diagnosing non-functioning tumors [11]. However, the literature states that the sensitivity of EUS-FNA in the diagnosis of PanNETs ranges widely, from $47 \%$ to $95 \%$ [12-14].
To overcome the limitations of EUS-FNA, fine-needle biopsy (FNB) has been developed for use with EUS scopes, to obtain core biopsies and improve histological diagnoses. The 19G Quick-Core (Cook UK, Limerick) needle (TNB) was first released, but despite its initial favorable results [15], more recent studies indicated that the overall efficacy profile of EUS-guided QuickCore needle biopsy appeared modest, with a reported diagnostic accuracy for pancreatic lesions of $33 \%$ to $61 \%[16,17]$. On the other hand, in a prospective study from our unit of $159 \mathrm{pa}$ tients [18], the combination of both EUS-FNA and Quick-Core sampling modalities resulted in higher diagnostic accuracy rates than either of the techniques alone, with reported diagnostic accuracies of pancreatic FNA, TNB and FNA/TNB of $77 \%$, $56 \%$, and $83 \%$, respectively. More recent FNB needles have been developed, such as the Procore (Cook UK, Limerick, Ireland) and the Sharkcore (Medtronic plc, Minneapolis, Minnesota, United States) needles, with encouraging results demonstrating the efficacy of EUS-guided core sampling [19-21].

We herein report and compare the results of different EUSguided sampling methods on patients in which the final diagnosis was that of a pancreatic neuroendocrine tumor.

\section{Patients and methods}

\section{Patients and procedures}

A retrospective review of a prospectively maintained database collected during a 13-year period (May 2004 - February 2017), at two tertiary hepato-pancreatico-biliary (HPB) endoscopy units that share the same endosonographers, the Royal Free Hospital and the University College London Hospital in London, was carried out. All clinical, endoscopic, and pathologic details 
of patients who underwent EUS-guided cell/tissue sampling of suspicious pancreatic lesions identified by pancreatic protocol CT or MRI were entered into the electronic database. All results, including technical aspects of the EUS procedures, were recorded.

EUS was performed under midazolam and fentanyl sedation, usually as a day-case procedure. All procedures were carried out by well-experienced endosonographers (>1000 procedures). Lesions underwent EUS-FNA or EUS-FNB sampling, or a combination of the two, employing different types of needles. Needle type and size, and number of passes, were decided at the discretion of the endoscopist.

All EUS-FNAs were performed using a disposable 22G or 25G FNA needle (EchoTip, Cook UK or Expect, Boston Scientific) in conjunction with linear-array echoendoscopes with a $2.8-$ $3.7 \mathrm{~mm}$ accessory channel (Olympus GF-UC240P-AL5 and GFUCT240-AL5, Keymed UK Ltd, Southend on Sea, UK). After visualization of the target lesion endosonographically, cell sampling was performed through the gastric or duodenal wall under real-time EUS guidance. The needle was visualized throughout the procedure. During EUS-FNA, the needle was moved back and forth during each pass, while applying suction using a $10-\mathrm{mL}$ syringe. Aspirated material was smeared onto glass slides and then alcohol fixed or air-dried, while possible aspirated fluid or material was placed into a specimen container.

EUS-FNB (Quick-Core, Cook UK, Procore, Cook UK or Sharkcore, Medtronic) was initially performed using the Quick-Core needles (Cook, UK) applying the same technique as reported previously [15-17]. Briefly, the needles used had a $22 \mathrm{G}$ or a 19 G outer cutting needle. Prior to use, the Quick-Core needle was primed by pulling back the spring-loaded handle assembly until it clicked into the "firing" position. This action drew the outer cutting needle back, allowing the inner needle with the tissue tray to be advanced into the target lesion under realtime EUS guidance, followed by firing of the spring-loaded cutting needle over the tray. The needle was then withdrawn back into the sheath and the entire assembly removed from the endoscope. Starting in 2011 , both centers were equipped with newly released FNB needles, such as the Procore (Cook UK) and Sharkcore (Medtronic) needles. Using the more recent FNB needles, first the lesions were visualized endosonographically and targeted with the needle. The needle was then moved back and forth during each pass, while applying suction with a 10-mL syringe. After sampling, the biopsied tissue from the needles was placed into a specimen container containing formalin. Adequacy of sampling by EUS-FNB was defined by successful acquisition of at least one core of tissue. There was no on-site microscopic evaluation of adequacy of EUS FNA or FNB material by a pathologist.

\section{Cytological and histological methods}

The pancreatic specimens examined included cytological material obtained from the EUS-FNA direct smears and liquid-based preparations and histological material obtained from the EUSFNA, formalin-fixed paraffin-embedded, cell-block, and core biopsy from EUS-FNB. When only cytological material was available, diagnosis of PanNET was based on compatible morpholo- gic findings of characteristic cytologic and nuclear features of the tumor cells, to include bland-appearing and monotonous cell morphology, round to oval nuclei and classical salt-andpepper chromatin distribution. However, cytological diagnosis was not conclusive in such cases as no confirmatory immunohistochemistry was possible and had to be integrated with clinical and radiological findings. Histologic diagnosis of PanNET was definitive when cell-block obtained from EUS-FNA and core biopsy obtained from EUS-FNB allowed immunohistochemical analysis demonstrating tumor cell positivity for cytokeratins and neuroendocrine markers (chromogranin and synaptophysin). Definitive diagnosis of PanNET was also provided when histological examination of surgical specimens was available.

Negative FNA/FNB results without any evidence of malignancy were confirmed as NETs during follow-up of at least 12 months by a multidisciplinary team specialized in neuroendocrine tumors (currently part of the ENET network), mainly based on a combination of clinical and imaging findings.

\section{Outcomes assessment}

The primary endpoint of the current study was diagnostic yield of various EUS-guided tissue sampling, defined as the proportion of cases in which an adequate specimen was obtained to pose a diagnosis, and assessment of variables possibly influencing diagnostic yield. Secondary outcomes included complication rates during the procedures and diagnostic sensitivity of various EUS-guided tissue sampling techniques, defined as the proportion of cases in which diagnosis of PanNET was posed among the adequate samples.

\section{Statistical analysis}

Continuous variables were described as median and interquartile range (IQR), whereas ordinal and categorical variables were reported as proportions. Comparisons between groups were performed with the Mann-Whitney $U$ test and Kruskal-Wallis test for continuous and ordinal variables. The Fisher's exact test and its extension, also known as Freeman-Halton test were used to compare categorical variables with two or more categories, respectively [22]. Diagnostic yield and diagnostic sensitivity were computed with $95 \% \mathrm{Cl}$, and considered significantly different between groups if $95 \% \mathrm{Cl}$ did not overlap. In this case, statistical significance was tested with Fisher's exact test for confirmation. Statistical analyses were performed with $\mathrm{R}$ version 3.4.2 for Mac [23].

\section{Results}

Of all patients who underwent EUS-FNA and/or EUS-FNB, 91 (M/F: 42/49) were diagnosed with PanNET after a total of 102 procedures, which was confirmed by multidisciplinary review and clinical follow-up. In 33 patients the diagnosis also was ascertained by additional histopathological examination of surgical resection specimens. Analysis of the technical aspects of pancreatic sampling during the 102 procedures revealed that both EUS-guided sampling modalities were used in 28 procedures, while EUS-FNA and EUS-FNB alone were used in 61 and 
- Table 1 Patient, lesion and sampling characteristics.

\begin{tabular}{|l|l|}
\hline Characteristics & $42 / 49$ \\
\hline - Gender (M/F) & $57(26-87)$ \\
\hline - Age, years (range) & $18(7-59)$ \\
\hline - Size of lesion, median mm (range) & \\
\hline Lesion diameter, group & $27(26.5 \%)$ \\
\hline - $\leq 10$ mm & $33(32.3 \%)$ \\
\hline - $>10$ mm and $\leq 20$ mm & $42(41.2 \%)$ \\
\hline - $>20$ mm & $34(33.3 \%)$ \\
\hline Lesion location, $n$ (\%) & $47(46.1 \%)$ \\
\hline - Pancreatic head & $21(20.6 \%)$ \\
\hline - Pancreatic body & \\
\hline - Pancreatic tail & $61(59.8 \%)$ \\
\hline Sampling technique & $13(12.7 \%)$ \\
\hline - FNA alone & $28(27.5 \%)$ \\
\hline - FNB alone & \\
\hline - FNA+ FNB & \\
\hline FNA, fine-needle aspiration; FNB, fine-needle biopsy & \\
\hline
\end{tabular}

13 cases, respectively. Patient and lesion characteristics are summarized in $>$ Table $\mathbf{1}$.

Target lesions were localized in the uncinate process/head of the pancreas in 34 cases, in the body in 47, while 21 were found in the tail. Median size of the lesions was $18 \mathrm{~mm}$ (range $7-$ $59 \mathrm{~mm}$ ). EUS-FNA was performed using a $22 \mathrm{G}$ needle in the majority of cases $74 / 89$ (83\%), while a $25 \mathrm{G}$ needle was used in the remaining $17 \%$. Median number of FNA needle passes was 3 (IQR 3 -4). To perform EUS-FNB, 23 (56\%) 19G Quick-Core, 14 (34\%) Procore (12 22G and 2 25G) and 4 (10\%) 22G Sharkcore needles were used. The median number of passes was three (IQR 3 -4) during the EUS-FNB procedures.

The diagnostic yield (i.e. obtaining an adequate sample) of EUS-FNA alone and EUS-FNB alone for the cytological/histological diagnosis was $77.5 \%(95 \% \mathrm{Cl}, 68.9-86.2 \%)$ and $85.4 \%(95 \%$ $\mathrm{Cl}, 74.6-96.2 \%)$, respectively. When the combination of both modalities was used, adequate sampling was achieved in 27 of 28 cases, with a diagnostic yield of $96.4 \%(95 \% \mathrm{Cl}, 89.6-100 \%)$, being significantly higher than EUS-FNA alone $(P=0.023)$. In particular, among the 28 cases, seven FNA specimens were insufficient for diagnosis, however, FNB samples were diagnostic for PanNET in six of them, while in one case neither of the two sampling modalities was diagnostic. Non-significant differences were observed when comparing performance of EUS-FNB needles: Quick-Core's diagnostic yield was $82.6 \%(95 \% \mathrm{Cl}$, 62.9-93\%), whereas the more recent Procore and Sharkcore needles had diagnostic yields of $85.7 \%(95 \% \mathrm{Cl}, 60.1-96 \%)$ and $100 \%(95 \% \mathrm{Cl}, 51-100 \%)$, respectively $(P=0.99$ for all of the comparisons).
No significant differences were observed for size and location of lesions, size of needles, or number of passes. $>$ Table 2 and Table 3 report the variables possibly influencing diagnostic yield of PanNET for FNA and FNB sampling.

Among the adequate samples, EUS-FNB specimens allowed a correct diagnosis of PanNET in 33 of 35 cases $(94.3 \%, 95 \% \mathrm{Cl}$, 86.6-100\%). In one case autoimmune pancreatitis was diagnosed and in another, no neuroendocrine tumor cells were seen. On the other hand, FNA samples were diagnostic in 61 of 69 cases $(88.4 \% ; 95 \% \mathrm{Cl}, 80.9-96.0 \%)$, in five cases no neuroendocrine tumor cells were found, two cases were suggestive for islet cell tumor and in one case pancreatitis was diagnosed. The combination of both sampling modalities allowed a correct diagnosis of PanNET in 27 of 27 cases $(100 \%$; $95 \% \mathrm{Cl}, 100-$ $100 \%)$.

Overall sensitivity of posing a correct diagnosis of PanNET based on the complete set of samples of EUS-FNA alone and EUS-FNB alone was $68.5 \%(95 \% \mathrm{Cl}, 58.9-78.2 \%)$ and $80.5 \%$ (95\% Cl, 68.4-92.6\%), respectively. The combination of both modalities achieved $96.4 \%(95 \% \mathrm{Cl}, 89.6-100 \%)$ overall sensitivity, significantly higher than EUS-FNA alone $(P=0.0021)$. There was only one reported complication where a patient developed a peripancreatic hematoma after EUS-FNA associated with $<2 \mathrm{~g} / \mathrm{dL}$ hemoglobin drop, and was admitted to the hospital for observation and discharged 2 days later without requiring blood transfusion or any further treatment.

\section{Discussion}

Preoperative diagnosis of PanNETs is important because it may alter clinical management [24], based on the unique biological nature of PanNETs and the wide range of therapeutic options available $[25,26]$. For example, in non-functioning PanNETs, patients often present with small pancreatic lesions on crosssectional imaging and preoperative histological confirmation could lead to pancreas-preserving surgery. EUS has shown to be superior to CT for detecting PanNETs, especially those $20 \mathrm{~mm}$. In a study from Khashab et al. [14], EUS detected $91 \%$ of CT-negative tumours, with a mean size of $18.8 \mathrm{~mm}$. Sensitivity of EUS was significantly greater than that for CT $(91.7 \%$ vs $63.3 \%)$ particularly for insulinomas.

EUS-FNA can provide cytological diagnosis of pancreatic lesions smaller than $10 \mathrm{~mm}$. However, the accuracy of the technique is largely dependent upon the size and location of the mass and expertise of the endoscopist [27]. EUS-FNA can also significantly increase the diagnostic impact in cases where PanNET were not identified after initial cross-sectional imaging [28]. Immediate review of the aspirated material by an experienced pathologist can improve adequacy of sampling as well as accuracy of cytological diagnosis [29], but this resource is not available in most institutions.

To date, there have been very few studies of EUS-FNA and of EUS-FNB in preoperative diagnosis of PanNETs. In two studies of 10 and 30 patients $[13,14]$, the accuracy of EUS-FNA was $90 \%$ and $83 \%$, respectively. In another two larger studies of 86 and 81 patients who had been diagnosed with PanNETs, $90 \%$ had the diagnosis established by EUS-FNA and the sensitivity was 
- Table 2 Exploratory analysis on variables possibly influencing EUS-FNA diagnostic yield.

\begin{tabular}{|c|c|c|c|}
\hline Lesion and sampling characteristics & Adequate sample on FNA $(n=69)$ & Inadequate sample on FNA $(n=20)$ & $P$ value \\
\hline \multicolumn{3}{|l|}{ Lesion site, n (\%) } & \multirow[t]{4}{*}{$0.789^{1}$} \\
\hline - Pancreatic head & $22(31.9 \%)$ & $8(40.0 \%)$ & \\
\hline - Pancreatic body & 35 (50.7\%) & $9(45.0 \%)$ & \\
\hline - Pancreatic tail & $12(17.4 \%)$ & $3(15.0 \%)$ & \\
\hline \multicolumn{3}{|l|}{ Lesion diameter, group } & \multirow[t]{4}{*}{$0.302^{2}$} \\
\hline . $\leq 10 \mathrm{~mm}$ & $22(31.9 \%)$ & $3(15.0 \%)$ & \\
\hline . $>10 \mathrm{~mm}$ and $\leq 20 \mathrm{~mm}$ & $20(29.0 \%)$ & $8(40.0 \%)$ & \\
\hline . $>20 \mathrm{~mm}$ & $27(39.1 \%)$ & $9(45.0 \%)$ & \\
\hline - Number of passes (median, IQR) & $3(3-4)$ & $3(2-4)$ & $0.101^{3}$ \\
\hline \multicolumn{3}{|l|}{ Size of needle, n (\%) } & \multirow[t]{3}{*}{$0.094^{4}$} \\
\hline $.22 \mathrm{G}$ & $60(87.0 \%)$ & $14(70.0 \%)$ & \\
\hline . $25 \mathrm{G}$ & $9(13.0 \%)$ & $6(30.0 \%)$ & \\
\hline \multicolumn{4}{|c|}{$\begin{array}{l}\text { EUS-FNA, endoscopic ultrasound-guided fine-needle aspiration; IQR, interquartile range } \\
{ }^{1} \text { Freeman-Halton test } \\
{ }^{2} \text { Kruskal-Wallis test } \\
{ }^{3} \text { Mann-Whitney U test } \\
{ }^{4} \text { Fisher's exact test }\end{array}$} \\
\hline
\end{tabular}

Table 3 Exploratory analysis of variables possibly influencing EUS-FNB diagnostic yield.

\begin{tabular}{|c|c|c|c|}
\hline Lesion and sampling characteristics & Adequate sample on FNB $(n=35)$ & Inadequate sample on FNB $(n=6)$ & $P$ value \\
\hline \multicolumn{3}{|l|}{ Lesion site, $\mathrm{n}(\%)$} & \multirow[t]{4}{*}{$0.163^{1}$} \\
\hline - Pancreatic head & $11(31.4 \%)$ & $4(66.7 \%)$ & \\
\hline - Pancreatic body & $14(40.0 \%)$ & $2(33.3 \%)$ & \\
\hline - Pancreatic tail & $10(28.6 \%)$ & $0(0 \%)$ & \\
\hline \multicolumn{3}{|l|}{ Lesion diameter, group } & \multirow[t]{4}{*}{$0.439^{2}$} \\
\hline - $\leq 10 \mathrm{~mm}$ & $4(11.4 \%)$ & $2(33.3 \%)$ & \\
\hline - $>10 \mathrm{~mm}$ and $\leq 20 \mathrm{~mm}$ & $10(28.6 \%)$ & $1(16.7 \%)$ & \\
\hline.$>20 \mathrm{~mm}$ & $21(60.0 \%)$ & $3(50.0 \%)$ & \\
\hline - Number of passes (median, IQR) & $3(2-4)$ & $3(2-4)$ & $0.566^{3}$ \\
\hline \multicolumn{3}{|l|}{ Type of needle, n (\%) } & \multirow[t]{4}{*}{$0.999^{1}$} \\
\hline $.19 \mathrm{G}$ & $19(57.1 \%)$ & $4(50 \%)$ & \\
\hline $.22 \mathrm{G}$ & $14(40.0 \%)$ & $2(33.3 \%)$ & \\
\hline $.25 \mathrm{G}$ & $2(2.9 \%)$ & $0(16.7 \%)$ & \\
\hline \multicolumn{3}{|l|}{ Size of needle, n (\%) } & \multirow[t]{4}{*}{$0.999^{1}$} \\
\hline - Quick-core & $19(54.3 \%)$ & $4(66.7 \%)$ & \\
\hline - ProCore & $12(34.3 \%)$ & $2(33.3 \%)$ & \\
\hline - SharkCore & $4(11.4 \%)$ & $0(0 \%)$ & \\
\hline \multicolumn{4}{|c|}{$\begin{array}{l}\text { EUS-FNB, endoscopic ultrasound-guided fine-needle biopsy; IQR, interquartile range } \\
{ }^{1} \text { Freeman-Halton test } \\
{ }^{2} \text { Kruskal-Wallis test } \\
{ }^{3} \text { Mann-Whitney U test }\end{array}$} \\
\hline
\end{tabular}


irrespective of tumor size or location [30,31]. In both studies, EUS-FNB was not performed. Pais et al. [32] reported a similar sensitivity for EUS-FNA in diagnosis of functional and non-functional PanNETs ( $77 \%$ and $91 \%$ respectively, $P=0.13$ ), but diminished sensitivity (66\%) for tumors $\leq 15 \mathrm{~mm}$. In an evaluation of solid pancreatic masses [33], accuracy of EUS-FNA in patients with PanNETs $(n=15)$ was significantly lower than that for adenocarcinomas $(n=59)(47 \%$ vs $81 \%, P<0.01)$. In contrast, in the current study, the diagnostic yield of EUS-FNA in the 89 PanNET-positive patients was $77.5 \%$, similar to our previously published data in patients with pancreatic adenocarcinoma [18, 34].

There are several possible explanations for the lower accuracy of EUS-FNA in PanNETs reported by other investigators. PanNETs are generally less than $2 \mathrm{~cm}$ in size [35], and it may be technically more difficult to obtain specimens from small lesions. Moreover, cytological features of PanNETs, unlike the much more common adenocarcinomas, are bland, resembling normal pancreatic acini. The most helpful morphological features that enable cytological diagnosis of PanNET are a richly cellular aspirate with a monotonous, poorly cohesive population of small cells with a speckled or dusty chromatin pattern and plasmacytoid morphology. The final diagnosis rests largely on confirmation of neuroendocrine differentiation of these cells by immunocytochemistry and can be very accurate [36, 37]. However, because PanNETs tend to be highly vascular, a bloody aspirate may dilute cellularity and relatively poorly cellular samples prevent application of further stains to confirm the diagnosis or grading of PanNETs; in a subset of patients in the current study, grading was available for 16 of 32 FNA cases ( $50 \%$ ) but 13 of 14 FNB cases (93\%).

To overcome these limitations, the EUS-FNB technique was proposed as a method that might replace or complement EUSFNA $[15,19-21]$. EUS-FNB devices have been designed to improve tissue yield and to potentially achieve a core histologic tissue specimen through EUS. However, lesions in the pancreatic head and uncinate process are particularly difficult to biopsy [16-18], in part because of their anatomical position and fibrotic nature, as well as due to needle rigidity, making at times trans-duodenal FNB puncture technically difficult. In PanNETs the tissue is not as fibrotic as in adenocarcinomas and especially for trans-gastric punctures, FNB is feasible. In the current series, six patients had their diagnosis established only by FNB because EUS-FNA did not establish a diagnosis because of inadequate $(n=5)$ or false-negative $(n=1)$ samples. Conversely, there were six patients with false-negative $(n=2)$ and inadequate $(n=$ 4) results with EUS-FNB (all cases performed with Quick-Core needles), whereas FNA established the diagnosis. Indeed, one limitation of EUS-FNB Quick-Core needle is the stiff firing mechanism and 2-cm tray of the needle, making it difficult to obtain tissue cores from small lesions in the head and uncinate process of the pancreas.

The more recent EUS-FNB devices have shown a higher diagnostic yield; smaller-diameter and more flexible biopsy FNB needles are now available and may improve tissue acquisition, particularly with a transduodenal approach [38]. In the study by Witt et al. the Sharkcore needles achieved a $90 \%$ diagnostic sensitivity with significantly fewer mean number of needle passes compared to EUS-FNA sampling, although the study included only 20 patients [20]. Our results confirm the high diagnostic yield of the EUS-FNB devices (85.4\%) for PanNET sampling, and also confirm that the combination of both FNA and FNB modalities may be helpful in selected patients, such as cases suspicious for PanNETs, to achieve a correct diagnoses [39].

The main limitation of this study is its retrospective design. Nevertheless, to date, the literature comparing diagnostic efficacy of different FNA and FNB needles in diagnosis of PanNETs has been limited. Most studies either include small study samples or compare the needles for heterogeneous cohorts of pancreatic lesions. The current study includes a reasonably large sample size, with more than 100 procedures, and focuses exclusively on PanNET cases.

\section{Conclusion}

In conclusion, EUS-FNA and EUS-FNB are useful and safe methods for tissue diagnosis in suspected PanNET. The addition of EUS-FNB needle biopsy improves the diagnostic yield from $77.5 \%$ for EUS-FNA alone to $96.4 \%$ for EUS-FNA + FNB, and thus provides additional clinical benefit to cytological assessment for preoperative diagnosis of PanNETs.

\section{Competing interests}

None

References

[1] Dasari A, Shen C, Halperin D et al. Trends in the incidence, prevalence, and survival outcomes in patients with neuroendocrine tumors in the United States. JAMA Oncol 2017; 3: 1335-1342

[2] Cives M, Strosberg JR. Gastroenteropancreatic neuroendocrine tumors. CA Cancer J Clin 2018; 68: 471-487

[3] Norton JA, Fraker DL, Alexander HR et al. Surgery to cure the Zollinger-Ellison syndrome. N Engl J Med 1999; 341: 635-644

[4] Wiedenmann B, Jensen RT, Mignon M et al. Preoperative diagnosis and surgical management of neuroendocrine gastroenteropancreatic tumors: general recommendations by a consensus workshop. World J Surg 1998; 22: 309-318

[5] Bombardieri E, Seregni E, Villano C et al. Position of nuclear medicine techniques in the diagnostic work-up of neuroendocrine tumors. Q J Nucl Med Mol Imaging 2004; 48: $150-163$

[6] Chiti A, Fanti S, Savelli G et al. Comparison of somatostatin receptor imaging, computed tomography and ultrasound in the clinical management of neuroendocrine gastro-entero-pancreatic tumours. Eur J Nucl Med 1998; 25: $1396-1403$

[7] Anderson MA, Carpenter S, Thompson NW et al. Endoscopic ultrasound is highly accurate and directs management in patients with neuroendocrine tumors of the pancreas. Am J Gastroenterol 2000; 95: $2271-2277$

[8] De Angelis C, Carucci P, Repici A et al. Endosonography in decision making and management of gastrointestinal endocrine tumors. Eur J Ultrasound 1999; 10: 139-150 
[9] Gouya H, Vignaux O, Augui J et al. CT, endoscopic sonography, and a combined protocol for preoperative evaluation of pancreatic insulinomas. AJR Am J Roentgenol 2003; 181: 987 -992

[10] Lee LS. Diagnosis of pancreatic neuroendocrine tumors and the role of endoscopic ultrasound. Gastroenterol Hepatol (N Y) 2010; 6: 520 522

[11] Metz DC, Jensen RT. Gastrointestinal neuroendocrine tumors: pancreatic endocrine tumors. Gastroenterology 2008; 135: 1469-1492

[12] Hijioka S, Hara K, Mizuno N et al. Diagnostic performance and factors influencing the accuracy of EUS-FNA of pancreatic neuroendocrine neoplasms. J Gastroenterol 2016; 51: $923-930$

[13] Ardengh JC, de Paulo GA, Ferrari AP. EUS-guided FNA in the diagnosis of pancreatic neuroendocrine tumors before surgery. Gastrointest Endosc 2004; 60: $378-384$

[14] Khashab MA, Yong E, Lennon AM et al. EUS is still superior to multidetector computerized tomography for detection of pancreatic neuroendocrine tumors. Gastrointest Endosc 2011; 73: 691 - 696

[15] Levy M], Jondal ML, Clain J et al. Preliminary experience with an EUSguided trucut biopsy needle compared with EUS-guided FNA. Gastrointest Endosc 2003; 57: 101 - 106

[16] Larghi A, Verna EC, Stavropoulos SN et al. EUS-guided Trucut needle biopsies in patients with solid pancreatic masses: a prospective study. Gastrointest Endosc 2004; 59: 185-190

[17] Varadarajulu S, Fraig M, Schmulewitz $\mathrm{N}$ et al. Comparison of EUSguided 19-gauge Trucut needle biopsy with EUS-guided fine-needle aspiration. Endoscopy 2004; 36: $397-401$

[18] Wittmann J, Kocjan G, Sgouros SN et al. Endoscopic ultrasound-guided tissue sampling by combined fine needle aspiration and trucut needle biopsy: a prospective study. Cytopathology 2006; 17: 27-33

[19] Fabbri C, Luigiano C, Maimone A et al. Endoscopic ultrasound-guided fine-needle biopsy of small solid pancreatic lesions using a 22-gauge needle with side fenestration. Surg Endosc 2015; 29: 1586 - 1590

[20] Witt BL, Factor RE, Chadwick BE et al. Evaluation of the SharkCore((R)) needle for EUS-guided core biopsy of pancreatic neuroendocrine tumors. Endosc Ultrasound 2018; 7: $322-328$

[21] Eusebi LH, Mogan S, Johnson G et al. High diagnostic accuracy and safety of the $22 \mathrm{~g}$ sharkcore needle for EUS-fine needle biopsy: a retrospective study from two tertiary centers in UK. Gastrointest Endosc 2017; 85: AB347-AB347

[22] Ruxton G, Neuhäuser M. Good practice in testing for an association in contingency tables. Behav Ecol Sociobiol 2010; 64: 1505-1513

[23] R Core Team. R: A language and environment for statistical computing. Vienna, Austria: R Foundation for Statistical Computing; 2017: ISBN 3-900051-07-0. http://www.R-project.org/

[24] Fritscher-Ravens A, Izbicki JR, Sriram PV et al. Endosonography-guided, fine-needle aspiration cytology extending the indication for organ-preserving pancreatic surgery. Am J Gastroenterol 2000; 95: $2255-2260$
[25] Ong SL, Garcea G, Pollard CA et al. A fuller understanding of pancreatic neuroendocrine tumours combined with aggressive management improves outcome. Pancreatology 2009; 9: 583-600

[26] Singh S, Dey C, Kennecke H et al. Consensus Recommendations for the Diagnosis and Management of Pancreatic Neuroendocrine Tumors: Guidelines from a Canadian National Expert Group. Ann Surg Oncol 2015; 22: 2685-2699

[27] Mallery JS, Centeno BA, Hahn PF et al. Pancreatic tissue sampling guided by EUS, CT/US, and surgery: a comparison of sensitivity and specificity. Gastrointest Endosc 2002; 56: 218 - 224

[28] Mitra V, Nayar MK, Leeds JS et al. Diagnostic performance of endoscopic ultrasound (EUS)/endoscopic ultrasound-fine needle aspiration (EUS-FNA) cytology in solid and cystic pancreatic neuroendocrine tumours. J Gastrointestin Liver Dis 2015; 24: 69-75

[29] Klapman JB, Logrono R, Dye CE et al. Clinical impact of on-site cytopathology interpretation on endoscopic ultrasound-guided fine needle aspiration. Am J Gastroenterol 2003; 98: 1289-1294

[30] Figueiredo FA, Giovannini M, Monges G et al. EUS-FNA predicts 5-year survival in pancreatic endocrine tumors. Gastrointest Endosc 2009; 70: $907-914$

[31] Atiq M, Bhutani MS, Bektas M et al. EUS-FNA for pancreatic neuroendocrine tumors: a tertiary cancer center experience. Dig Dis Sci 2012; 57: $791-800$

[32] Pais SA, Al-Haddad M, Mohamadnejad M et al. EUS for pancreatic neuroendocrine tumors: a single-center, 11 -year experience. Gastrointest Endosc 2010; 71: 1185-1193

[33] Voss M, Hammel P, Molas G et al. Value of endoscopic ultrasound guided fine needle aspiration biopsy in the diagnosis of solid pancreatic masses. Gut 2000; 46: 244-249

[34] Aithal GP, Anagnostopoulos GK, Tam W et al. EUS-guided tissue sampling: comparison of "dual sampling" (Trucut biopsy plus FNA) with "sequential sampling" (Trucut biopsy and then FNA as required). Endoscopy 2007; 39: $725-730$

[35] Jani N, Khalid A, Kaushik N et al. EUS-guided FNA diagnosis of pancreatic endocrine tumors: new trends identified. Gastrointest Endosc 2008; 67: $44-50$

[36] Chang F, Vu C, Chandra A et al. Endoscopic ultrasound-guided fine needle aspiration cytology of pancreatic neuroendocrine tumours: cytomorphological and immunocytochemical evaluation. Cytopathology 2006; 17: $10-17$

[37] Chatzipantelis P, Salla C, Konstantinou P et al. Endoscopic ultrasoundguided fine-needle aspiration cytology of pancreatic neuroendocrine tumors: a study of 48 cases. Cancer 2008; 114: 255-262

[38] Iglesias-Garcia J, Poley JW, Larghi A et al. Feasibility and yield of a new EUS histology needle: results from a multicenter, pooled, cohort study. Gastrointest Endosc 2011; 73: 1189-1196

[39] Hedenstrom P, Demir A, Khodakaram K et al. EUS-guided reverse bevel fine-needle biopsy sampling and open tip fine-needle aspiration in solid pancreatic lesions - a prospective, comparative study. Scand J Gastroenterol 2018; 53: 231-237 